\title{
Duality for Dual Covariance Algebras
}

\author{
Magnus B. Landstad \\ Matematisk Institutt, Universitetet i Trondheim, Norges Laererhøgskole, N-7000 Trondheim, Norway
}

\begin{abstract}
One way of generalizing the definition of an action of the dual group of a locally compact abelian group on a von Neumann algebra to nonabelian groups is to consider $\mathscr{L}(G)$-comodules, where $\mathscr{L}(G)$ is the Hopfvon Neumann algebra generated by the left regular representation of $G$. To a $\mathscr{L}(G)$-comodule we shall associate a dual covariance algebra $\mathfrak{A}$ and a natural covariant system $(\mathfrak{U}, \varrho, G)$, and in Theorem 1 the covariant systems coming from $\mathscr{L}(G)$-comodules are characterized. In [2] it was shown that the covariance algebra of a covariant system in a natural way is a $\mathscr{L}(G)$-comodule. Therefore one can form the dual covariance algebra of a covariance algebra and the covariance algebra of a dual covariance algebra. Theorems 2 and 3 deal with these algebras - generalizing a result by Takesaki. As an application we give a new proof of a theorem by Digernes stating that the commutant of a covariance algebra itself is a covariance algebra and prove the similar result for dual covariance algebras.
\end{abstract}

\section{§1. Introduction}

If $G$ is a locally compact group and $\varrho: G \rightarrow \operatorname{Aut}(A)$ is a continuous homomorphism of $G$ into the group of *-automorphisms of a von Neumann algebra $A$, $(A, \varrho, G)$ is called a covariant system and one can form the covariance algebra $\mathfrak{A}=W^{*}(A, \varrho, G)$. Takesaki showed in [8] that if $G$ is abelian there is a natural covariant system $\left(\mathfrak{A}, \tau, G^{\wedge}\right)$ over the dual group $G^{\wedge}$ and that $W^{*}\left(\mathfrak{A}, \tau, G^{\wedge}\right) \cong$ $A \otimes \mathscr{B}\left(L^{2}(G)\right.$ ), i.e. the tensorproduct of $A$ with the algebra of all bounded operators on $L^{2}(G)$.

For a non-abelian $G$ there is no dual group to act on the covariance algebra $\mathfrak{U}=W^{*}(A, \varrho, G)$, but this author showed in [2] that the natural structure on $\mathfrak{A}$ corresponding to the action of a dual group is that of a $\mathscr{L}(G)$-comodule. There one used that $\mathscr{L}(G)$, the von Neumann algebra generated by the left regular representation of $G$ is a Hopf-von Neumann algebra, cf. [7].

So if $A$ is a von Neumann algebra, what seems to correspond to a covariant system on $A$ over $G^{\wedge}$ if $G$ is abelian is that of a $\mathscr{L}(G)$-comodule structure on $A$. 
Given such a comodule we shall define a corresponding dual covariance algebra $\mathfrak{A}$, and it turns out that there is a natural covariant system $(\mathfrak{Q}, \varrho, G)$ over $\mathfrak{A}$. In Theorem 1 we characterize those covariant systems $(\mathfrak{A}, \varrho, G)$ which come from $\mathscr{L}(G)$-comodules, this is a dual version of Theorem 1 in [2].

Since the covariance algebra $\mathfrak{U}$ of a covariant system is a $\mathscr{L}(G)$-comodule it is natural to ask what the dual covariance algebra of $\mathfrak{Q}$ is, furthermore if $\mathfrak{A}$ is the dual covariance algebra of a $\mathscr{L}(G)$-comodule and $(\mathfrak{A}, \varrho, G)$ the corresponding covariant system one wants to know what the covariance algebra of $(\mathfrak{U}, \varrho, G)$ is. The answers to these two questions are given in Theorems 2 and 3 and are natural generalizations of Takesaki's result ([8, Theorem 4.5]) mentioned above.

Finally as an application of Theorem 1 in [2] and Theorem 1 in this article we prove that

(a) the commutant of a covariance algebra over $G$ is itself a covariance algebra over $G$, and

(b) the commutant of a dual covariance algebra over $G$ is itself a dual covariance algebra over $G$.

Digernes has proved (a) by other methods in [1].

Roberts has given a different, but related definition of an action of the dual of a group on a von Neumann algebra in [5]. His dual objects are sets of representations of $G$, while we in this article exploit the duality between the Hopf-von Neumann algebras $\mathscr{L}(G)$ and $L^{\infty}(G)$ developed in [7].

A similar notion of dual action has been given by Nakagami, [ 3 and 4]. His main tool is a very interesting sort of Fourier analysis on the Hopf-von Neumann algebra $\mathscr{L}(G)$ [or rather $\left.\mathscr{L}(G)^{\prime}\right]$.

The author wants to thank Professor M. Takesaki for making him aware of Nakagami's work. In the final stage of this work the author also received a preprint [6] by S. Strătilă, D. Voiculescu and L. Zsidó announcing the same results.

\section{§2. Duality for Dual Crossed Products}

If $G$ is a locally compact group we shall always equip $G$ with a left invariant Haarmeasure, usually denoted $d x . \Delta$ is the modular function on $G$. The spaces $L^{p}(G)$ with norm \|\|$_{p}(1 \leqq p \leqq \infty)$ are always with respect to this measure. $C_{00}(G)$ is the space of all continuous complex valued functions on $G$ with compact support. $\mathscr{L}(G)$ is the von Neumann algebra generated by the left regular representation of $G$, and for $x \in G$ we shall denote with $x$ also the corresponding element of $\mathscr{L}(G)$, i.e.

$$
(x f)(y)=f\left(x^{-1} y\right) \text { for } \quad x, y \in G, f \in L^{2}(G) \text {. }
$$

$\mathscr{R}(G)=\mathscr{L}(G)^{\prime}$ is generated by $\{v(x) \mid x \in G\}$ where

$$
(v(x) f)(y)=\Delta(x)^{1 / 2} f(y x) \text { for } x, y \in G, f \in L^{2}(G) .
$$

If $\varphi \in L^{\infty}(G)$, we shall also denote with $\varphi$ the corresponding operator on $L^{2}(G)$, i.e.

$$
(\varphi f)(x)=\varphi(x) f(x) \text { for } \varphi \in L^{\infty}(G), f \in L^{2}(G) .
$$

If $H$ is a Hilbert space, $\mathscr{B}(H)$ is the von Neumann algebra of all bounded operators on $H$. 
If $W_{G}$ is the unitary operator over $L^{2}(G \times G)$ defined by

$$
W_{G} f(s, t)=f(s, s t) \text { for } s, t \in G, f \in L^{2}(G \times G),
$$

we can define a normal isomorphism $\delta_{G}: \mathscr{L}(G) \rightarrow \mathscr{L}(G) \otimes \mathscr{L}(G)$ by

$$
\delta_{G}(a)=W_{G}^{*}(a \otimes I) W_{G} \quad \text { for } \quad a \in \mathscr{L}(G) .
$$

$A(G)=\mathscr{L}(G)_{*}=$ the predual of $\mathscr{L}(G)$ is an algebra under the multiplication defined by

$$
\alpha \beta(a)=(\alpha \otimes \beta)\left(\delta_{G}(a)\right) \text { for } \quad a \in \mathscr{L}(G), \alpha, \beta \in A(G) .
$$

In fact $A(G)$ will be a commutative semi-simple Banach *-algebra and is usually called the Fourier-algebra of $G$, cf. $[7, \S 2]$. For any unexplained definitions and notations concerning von Neumann algebras and covariant systems we refer to [2].

Suppose now $A$ is a von Neumann algebra realized over some Hilbert space $H_{0}$. If we have a normal isomorphism $\delta: A \rightarrow A \otimes \mathscr{L}(G)$ satisfying

$(\delta \otimes i) \delta=\left(i \otimes \delta_{\mathrm{G}}\right) \delta$

we shall call the pair $(A, \delta)$ a $\mathscr{L}(G)$-comodule, and we say that $\delta$ is a dual action of $G$ on $A$. (Note that if $G$ is abelian (7) will in fact define a covariant system for $A$ and $G^{\wedge}$, cf. [4, Theorem 2.1].) As noted in [2] a comodule $(A, \delta)$ will make the predual $A_{*}$ of $A$ into an $A(G)$-module if we define

$$
\varphi \alpha(a)=(\varphi \otimes \alpha)(\delta(a)) \quad \text { for } \quad \varphi \in A_{*}, \alpha \in A(G), a \in A .
$$

Definition. Let $\mathfrak{A}=W^{*}(A, \delta, G)$ be the von Neumann algebra generated by $\delta(A) \cup I \otimes L^{\infty}(G)$ over $H=L^{2}\left(G, H_{0}\right) \cong H_{0} \otimes L^{2}(G)$. $\mathfrak{A}$ is called the dual covariance algebra of $(A, \delta)$ and does not depend on the Hilbert space $H_{0}$ on which $A$ is represented.

Let $\mu: L^{\infty}(G) \rightarrow \mathfrak{A}$ be the normal isomorphism defined by $\mu(f)=I \otimes f$. We can define a $\sigma$-continuous automorphic representation $\varrho$ of $G$ on $\mathfrak{A}$ by the formula

$$
\varrho_{x}(a)=(I \otimes v(x)) a\left(I \otimes v\left(x^{-1}\right)\right) \quad \text { for } \quad x \in G, a \in \mathfrak{A} .
$$

Then $\varrho_{x}(\delta(a))=\delta(a)$ for $a \in A$ and $\varrho_{x}(\mu(f))=\mu\left(f_{x}\right)$ for $f \in L^{\infty}(G)$ where

$$
f_{x}(y)=f(y x) \text {. }
$$

So we have that $\varrho_{x}(\mathfrak{A})=\mathfrak{A}$, and $(\mathfrak{A}, \varrho, G)$ is in fact a covariant system.

Therefore if $\mathfrak{A}=W^{*}(A, \delta, G)$ is the dual covariance algebra of a $\mathscr{L}(G)$-comodule $(A, \delta)$ we can define a $\sigma$-continuous automorphic representation $\varrho: G \rightarrow \operatorname{Aut}(\mathfrak{R})$ and a normal isomorphism $\mu: L^{\infty}(G) \rightarrow \mathfrak{A}$ such that

$$
\varrho_{x}(\mu(f))=\mu\left(f_{x}\right) \text { for } f \in L^{\infty}(G), x \in G .
$$

Our main result is that this property in fact characterizes such dual covariance algebras:

Theorem 1. Given a von Neumann algebra $\mathfrak{A}$ and a locally compact group $G$, then $\mathfrak{A}$ is the dual covariance algebra of some $\mathscr{L}(G)$-comodule $(A, \delta)$ if and only if there is a $\sigma$-continuous automorphic representation @ of $G$ over $\mathfrak{A}$ and a normal isomorphism 
$\mu$ of $L^{\infty}(G)$ into $\mathfrak{U}$ such that

$$
\varrho_{x}(\mu(f))=\mu\left(f_{x}\right) \quad \text { for } f \in L^{\infty}(G), x \in G .
$$

$(A, \delta)$ are uniquely determined up to isomorphisms by

$$
A \cong\left\{a \in \mathfrak{A} \mid \varrho_{x}(a)=a \text { for all } x \in G\right\}
$$

and

$$
\langle\delta(a)(\xi \otimes f), \eta \otimes g\rangle=\int\left\langle a \mu\left(f_{t}\right) \xi, \mu\left(g_{t}\right) \eta\right\rangle d t
$$

for $a \in A, \xi, \eta \in H, f, g \in C_{00}(G)$, if $\mathfrak{A}$ is considered as a von Neumann algebra over a Hilbert space $H$.

The first step is to prove the uniqueness above, in fact we shall prove:

Lemma 1. If $\mathfrak{U}=W^{*}(A, \delta, G)$ and $\varrho$ is defined by (9), then

$$
\delta(A)=\left\{a \in \mathfrak{A} \mid \varrho_{x}(a)=a \quad \text { for all } \quad x \in G\right\} .
$$

Proof. Let $B$ be the right hand side of (14), so $B=\mathfrak{A} \cap(I \otimes \mathscr{R}(G))^{\prime}$. Obviously $\delta(A) \subset B$. Since $\delta(A) \subset A \otimes \mathscr{L}(G)$ we have

$$
A^{\prime} \otimes \mathscr{R}(G) \subset \delta(A)^{\prime} .
$$

Let $W=I \otimes W_{G}$, so $W$ is a unitary operator over $H \otimes L^{2}(G) \cong H_{0} \otimes L^{2}(G \times G)$. Then

$$
\begin{aligned}
& W^{*}(\delta(A) \otimes I) W=\left(i \otimes \delta_{G}\right) \delta(A)=(\delta \otimes i) \delta(A) \\
& C(\delta \otimes i)(A \otimes \mathscr{L}(G)) .
\end{aligned}
$$

So $\delta(A)^{\prime} \otimes \mathscr{R}(G) \subset W^{*}\left(\delta(A)^{\prime} \otimes \mathscr{B}\left(L^{2}(G)\right) W\right.$. Since $W \in(I \otimes I \otimes \mathscr{R}(G))^{\prime}$, this implies that $W\left(\delta(A)^{\prime} \otimes I\right) W^{*} \subset \delta(A)^{\prime} \otimes \mathscr{L}(G)$. Therefore we can define a normal isomorphism $\delta^{\prime}: \delta(A)^{\prime} \rightarrow \delta(A)^{\prime} \otimes \mathscr{L}(G)$ by

$$
\delta^{\prime}(a)=W(a \otimes I) W^{*} \quad \text { for } \quad a \in \delta(A)^{\prime} .
$$

By $(15) I \otimes v(x) \in \delta(A)^{\prime}$ for $x \in G$, and

$$
\delta^{\prime}(I \otimes v(x))=I \otimes v(x) \otimes x \quad \text { for } \quad x \in G .
$$

We next want to prove that $\left(\delta^{\prime} \otimes i\right) \delta^{\prime}=\left(i \otimes \delta_{G}\right) \delta^{\prime}$. If we extend the definition of $\delta^{\prime}$ to all elements in $\mathscr{B}(H)$ using the same formula (16), it will suffice to prove that

$$
\left(\delta^{\prime} \otimes i\right) \delta^{\prime}(a \otimes f \cdot v(x))=\left(i \otimes \delta_{G}\right) \delta^{\prime}(a \otimes f \cdot v(x))
$$

for all $a \in \mathscr{B}\left(H_{0}\right), f \in L^{\infty}(G), x \in G$, since elements of this form is a total set in $\mathscr{B}(H)$. Now if we use (17) and that $W \in\left(I \otimes L^{\infty}(G) \otimes I\right)^{\prime}$ we have

$$
\delta^{\prime}(a \otimes f \cdot v(x))=W(a \otimes f \cdot v(x) \otimes I) W^{*}=a \otimes f \cdot v(x) \otimes x .
$$

So the left hand side of (18) equals

$$
\begin{aligned}
& \left(\delta^{\prime} \otimes i\right)(a \otimes f \cdot v(x) \otimes x)=a \otimes f \cdot v(x) \otimes x \otimes x=a \otimes f \cdot v(x) \otimes \delta_{G}(x) \\
& =\left(i \otimes \delta_{G}\right)(a \otimes f \cdot v(x) \otimes x)=\text { right hand side of }(18) .
\end{aligned}
$$


This means that $\left(\delta(A)^{\prime}, \delta^{\prime}\right)$ is a $\mathscr{L}(G)$-comodule and that the map $I \otimes v: G \rightarrow \delta(A)^{\prime}$ is such that the triple $\left(\delta(A)^{\prime}, I \otimes v, \delta^{\prime}\right)$ satisfies the assumptions of [2, Theorem 1] so we have in particular that

$$
\delta(A)^{\prime}=[C \cup I \otimes \mathscr{R}(G)]^{\prime \prime}
$$

where

$$
C=\left\{c \in \delta(A)^{\prime} \mid W(c \otimes I) W^{*}=c \otimes I\right\} .
$$

Now in order to prove that $\delta(A)=B$ it suffices to show that $\delta(A)^{\prime} \subset B^{\prime}$, i.e. that $C \cup I \otimes \mathscr{R}(G) \subset B^{\prime}$. Obviously $I \otimes \mathscr{R}(G) \subset B^{\prime}$, so we must show that $C \subset B^{\prime}$. Since $\mathfrak{U}^{\prime} \subset B^{\prime}$, it suffices to show that

$$
C \subset \mathfrak{U}^{\prime}=\delta(A)^{\prime} \cap\left(I \otimes L^{\infty}(G)\right)^{\prime} \text {, i.e. that } \quad C \subset\left(I \otimes L^{\infty}(G)\right)^{\prime} .
$$

This follows from observing that if $c \in C$, then

$$
\begin{aligned}
c \otimes I & =W(c \otimes I) W^{*} \in W\left(I \otimes I \otimes L^{\infty}(G)\right)^{\prime} W^{*} \cap\left(I \otimes I \otimes L^{\infty}(G)\right)^{\prime} \\
& =\left(I \otimes L^{\infty}(G) \otimes L^{\infty}(G)\right)^{\prime} .
\end{aligned}
$$

So $c \in\left(I \otimes L^{\infty}(G)\right)^{\prime}$, in fact $C=\delta(A)^{\prime} \cap\left(I \otimes L^{\infty}(G)\right)^{\prime}$. This proves the lemma.

We have now proved that if we start with a $\mathscr{L}(G)$-comodule $(A, \delta)$ and $\mathfrak{A}=$ $W^{*}(A, \delta, G)$ we recover $(A, \delta)$ or rather the isomorphic comodule $(\delta(A), \delta \otimes i)$ as follows: $\delta(A)=\left\{a \in \mathfrak{A} \mid \varrho_{x}(a)=a\right.$ for all $\left.x \in G\right\}$. That $\delta \otimes i$ then in fact is given by the formula (13) follows from:

Lemma 2. If $a \in \mathscr{B}\left(H_{0}\right) \otimes \mathscr{L}(G), f, g \in C_{00}(G), \xi, \eta \in L^{2}\left(G, H_{0}\right)$ we have that

$$
\left\langle W^{*}(a \otimes I) W(\xi \otimes f), \eta \otimes g\right\rangle=\int\left\langle a \mu\left(f_{t}\right) \xi, \mu\left(g_{t}\right) \eta\right\rangle d t .
$$

Proof. It suffices to prove this for $a=b \otimes x$ with $b \in \mathscr{B}\left(H_{0}\right), x \in G$, since both sides define bounded normal linear functionals on $\mathscr{B}(H)$.

$$
\begin{aligned}
& \left\langle W^{*}(b \otimes x \otimes I) W(\xi \otimes f), \eta \otimes g\right\rangle=\langle(b \otimes x \otimes x)(\xi \otimes f), \eta \otimes g\rangle \\
& =\langle x f, g\rangle\langle(b \otimes x) \xi, \eta\rangle=\iint f\left(x^{-1} t\right) \overline{g(t)}\left\langle b \xi\left(x^{-1} s\right), \eta(s)\right\rangle d t d s \\
& =\iint f\left(x^{-1} s t\right) \overline{g(s t)}\left\langle b \xi\left(x^{-1} s\right), \eta(s)\right\rangle d s d t \\
& =\iint\left\langle b\left(\mu\left(f_{t}\right) \xi\right)\left(x^{-1} s\right),\left(\mu\left(g_{t}\right) \eta\right)(s)\right\rangle d s d t \\
& =\int\left\langle(b \otimes x) \mu\left(f_{t}\right) \xi, \mu\left(g_{t}\right) \eta\right\rangle d t .
\end{aligned}
$$

Let us now turn to the second part of Theorem 1, so suppose we have a von Neumann algebra $\mathfrak{A}$ over a Hilbert space $H, \varrho: G \rightarrow \operatorname{Aut}(\mathfrak{U})$ and $\mu: L^{\infty}(G) \rightarrow \mathfrak{U}$ as in Theorem 1. We want to prove that $\mathfrak{A} \cong W^{*}(A, \delta, G)$ where $(A, \delta)$ is defined by (12) and (13). Let

$$
\mathfrak{U}_{0}=\left\{a \in \mathfrak{U} \mid \exists K \geqq 0, \int \varphi \circ \varrho_{x}\left(a^{*} a\right) d x \leqq K\|\varphi\| \text { for all } \varphi \in \mathfrak{U}_{*}^{+}\right\} .
$$

Then $\mathfrak{A P}_{0} \subset \mathfrak{U}_{0}$ and $\mathfrak{I}_{1}=$ the *-algebra generated by $\mathfrak{H}_{0}^{*} \mathfrak{U}_{0}$ is a ${ }^{*}$-subalgebra of $\mathfrak{U}$.

Lemma 3. $\mathfrak{U}_{0}$ and $\mathfrak{U}_{1}$ are both $\sigma$-dense in $\mathfrak{U}$.

Proof. Since there is a net $f_{i} \in C_{00}(G)$ such that $\mu\left(f_{i}\right) \rightarrow I$ in the $\sigma$-topology and since $\mathfrak{U}_{0}$ is a left ideal in $\mathfrak{U}$, it suffices to prove that $\mu(f) \in \mathfrak{U}_{0}$ for all $f \in C_{00}(G)$. 
If $\varphi \in \mathfrak{A}_{*}^{+}, \varphi \circ \mu \in L^{\infty}(G)_{*}^{+} \cong L^{1}(G)^{+}$, so there is a function $h \in L^{1}(G)^{+}$such that $\varphi \circ \mu(f)=\int f(x) h(x) d x$ for all $f \in L^{\infty}(G)$.

If $f \in C_{00}(G)$ we therefore have:

$$
\begin{aligned}
& \int \varphi \circ \varrho_{x}\left(\mu\left(f^{*} f\right)\right) d x=\int \varphi \circ \mu\left(\left(f^{*} f\right)_{x}\right) d x \\
& =\iint\left(f^{*} f\right)_{x}(y) h(y) d x d y=\iint|f(y x)|^{2} h(y) d x d y \\
& =\int|f(x)|^{2} d x \int h(y) d y=\|f\|_{2}^{2} \varphi(I)=\|f\|_{2}^{2}\|\varphi\|,
\end{aligned}
$$

since there are no problems in changing the order of integration. So $\mu(f) \in \mathfrak{A}_{0}$.

Now let $A=\left\{a \in \mathfrak{U} \mid \varrho_{x}(a)=a\right.$ for all $\left.x \in G\right\}$ and define a positive linear map $p: \mathfrak{U}_{1} \rightarrow A$ by requiring

$$
\varphi(p(a))=\int \varphi \circ \varrho_{x}(a) d x \text { for all } \varphi \in \mathfrak{A}_{*} .
$$

Note that we in Lemma 3 in fact proved that for $f \in C_{00}(G)$

$$
p\left(\mu\left(f^{*} f\right)\right)=\|f\|_{2}^{2} I .
$$

Lemma 4. $A$ and $\mu\left(L^{\infty}(G)\right)$ generates $\mathfrak{A}$.

Proof. Let $\mathscr{B}=\left[A \cup \mu\left(L^{\infty}(G)\right)\right]^{\prime \prime}$, we shall prove that $\mathfrak{A}=\mathscr{B}$. It will by Lemma 3 suffice to show that each element of the form $b=a \mu(\alpha)$ is in $\mathscr{B}$ for all $a \in \mathfrak{U}$ and $\alpha \in C_{00}(G)$. So suppose such an element $b=a \mu(\alpha)$ is given together with a $\varphi \in \mathfrak{A}_{*}$ and $\varepsilon>0$. Choose a compact neighbourhood $U$ of $e$ in $G$ such that

$$
\left|\varphi\left(\varrho_{y}(b)-b\right)\right|<\varepsilon \text { for all } y \in U .
$$

Take another neighbourhood $V$ of $e$ with $V^{-1} V \subset U$ and functions $f, g \in C_{00}(G)^{+}$ with supports in $V$ and such that

$$
\int f(y) d y=\int g\left(x^{-1}\right) d x=1 .
$$

Take $h(y)=\int f\left(x^{-1} y\right) g\left(x^{-1}\right) d x$, then $h$ has support in $U$ and $\int h(y) d y=1$. Let ${ }_{x} f(y)=f\left(x^{-1} y\right)$ and define an element $c$ by

$$
\begin{aligned}
c & =\int p\left[b \mu\left({ }_{x} f\right)\right] \mu\left({ }_{x} g\right) d x \\
& =\iint \varrho_{y}(a) \mu\left(\alpha_{y} \cdot{ }_{x} f_{y} \cdot{ }_{x} g\right) d y d x .
\end{aligned}
$$

If $K=\operatorname{support}(\alpha)$ we have that $\left(\alpha_{y}{ }_{x} f_{y} \cdot{ }_{x} g\right)(z)=\alpha(z y) f\left(x^{-1} z y\right) g\left(x^{-1} z\right) \neq 0$ only if $x \in K V^{-1}$ and $y \in V^{-1} V$. So $c$ is well-defined as a weak Bochner integral and $c \in \mathscr{B}$.

$$
\int f\left(x^{-1} z y\right) g\left(x^{-1} z\right) d x=\int f\left(x^{-1} y\right) g\left(x^{-1}\right) d x=h(y)
$$

for all $z \in G$, so $\int \mu\left({ }_{x} f_{y} \cdot{ }_{x} g\right) d x=h(y) I$. By changing the order of integration in (25) we see that

$$
c=\int h(y) \varrho_{y}(b) d y .
$$

Therefore

$$
\begin{aligned}
& |\varphi(c-b)|=\left|\int h(y) \varphi\left(\varrho_{y}(b)-b\right) d y\right| \\
& \leqq \int_{U} h(y)\left|\varphi\left(\varrho_{y}(b)-b\right)\right| d y \leqq \varepsilon \int h(y) d y=\varepsilon .
\end{aligned}
$$


So $\mathfrak{A} \mu\left(C_{00}(G)\right) \subset \mathscr{B}$, thus $\mathscr{B}$ is $\sigma$-dense in $\mathfrak{A}$, and $\mathfrak{A}=\mathscr{B}$.

We now want to make $A$ into a $\mathscr{L}(G)$-comodule and for $a \in \mathfrak{A}$ we shall define an element $\delta(a)$ of $\mathscr{B}\left(L^{2}(G, H)\right)$ by

$$
\langle\delta(a)(\xi \otimes f), \eta \otimes g\rangle=\int\left\langle\varrho_{t}(a) \mu\left(f_{t}\right) \xi, \mu\left(g_{t}\right) \eta\right\rangle d t
$$

for $\xi, \eta \in H, f, g \in C_{00}(G)$.

Note that for $a \in A$ the definitions (27) and (13) agree. First let us check that (27) really defines a bounded operator. If $a \in \mathfrak{U},\left\{f^{i}\right\}_{i=1}^{n} \subset C_{00}(G)$ and $\left\{\xi^{i}\right\}_{i=1}^{n} \subset H$ we have by (23) that

$$
\begin{aligned}
& \int\left\|\sum_{i} \varrho_{t}(a) \mu\left(f_{t}^{i}\right) \xi^{i}\right\|^{2} d t \leqq\|a\|^{2} \sum_{i, j}\left\langle\mu\left(f_{t}^{i}\right) \xi^{i}, \mu\left(f_{t}^{j}\right) \xi^{j}\right\rangle d t \\
& =\|a\|^{2} \sum_{i, j}\left\langle f^{i}, f^{j}\right\rangle\left\langle\xi^{i}, \xi^{j}\right\rangle=\|a\|^{2}\left\|\sum_{i} f^{i} \otimes \xi^{i}\right\|^{2} .
\end{aligned}
$$

So (27) really defines $\delta(a)$ as a bounded operator over $L^{2}(G, H)$.

Obviously $\delta(a) \in \mathfrak{A} \otimes \mathscr{B}\left(L^{2}(G)\right)$ for $a \in \mathfrak{U}$. Furthermore, if $a \in \mathfrak{U}$

$$
\begin{aligned}
& \left\langle\left(I \otimes v(x) \delta(a)\left(I \otimes v\left(x^{-1}\right)\right)(\xi \otimes f), \eta \otimes g\right\rangle\right. \\
& =\left\langle\delta(a)\left(\xi \otimes v\left(x^{-1}\right) f\right), \eta \otimes v\left(x^{-1}\right) g\right\rangle \\
& =\Delta\left(x^{-1}\right) \int\left\langle\varrho_{t}(a) \mu\left(f_{t x^{-1}}\right) \xi, \mu\left(g_{t x^{-1}}\right) \eta\right\rangle d t \\
& =\left\langle\delta\left(\varrho_{x}(a)\right)(\xi \otimes f), \eta \otimes g\right\rangle .
\end{aligned}
$$

So $\delta(A) \subset \mathfrak{U} \otimes \mathscr{L}(G)$.

If $\varphi \in \mathfrak{U}_{*}, f, g \in C_{00}(G)$ and $h \in A(G)$ is defined by $h(x)=\langle x \cdot f, g\rangle$ for $x \in \mathscr{L}(G)$ we have from (27) that

$$
\begin{aligned}
& (\varphi \otimes h) \circ\left(\varrho_{x} \otimes i\right)(\delta(a))=\left(\varphi \circ \varrho_{x} \otimes h\right)(\delta(a)) \\
& =\int \varphi \circ \varrho_{x}\left(\mu\left(g_{t}\right) * a \mu\left(f_{t}\right)\right) d t \\
& =\int \varphi\left(\mu\left(g_{x t}\right) * a \mu\left(f_{x t}\right)\right) d t=(\varphi \otimes h)(\delta(a))
\end{aligned}
$$

for all $a \in A, x \in G$. Thus $\delta(A) \subset A \otimes \mathscr{L}(G)$.

Now note that from (23) it follows that we can define a unitary operator $U$ over $L^{2}(G, H)$ such that

$$
U(\xi \otimes f)(s)=\mu\left(f_{s}\right) \xi \quad \text { for } \quad \xi \in H, f \in C_{00}(G) .
$$

If $\varrho_{1}$ is the normal isomorphism of $\mathfrak{U}$ defined by

$$
\varrho_{1}(a) f(s)=\varrho_{s}(a) f(s) \text { for } a \in A, f \in L^{2}(G, H), s \in G,
$$

we see from (27) that we have

$$
\delta(a)=U^{*} \varrho_{1}(a) U \text { for } \quad a \in \mathfrak{A},
$$

so $\delta$ is obviously a normal isomorphism. 
In order to show that $(A, \delta)$ is a $\mathscr{L}(G)$-comodule it now remains to show that $(\delta \otimes i) \delta=\left(i \otimes \delta_{G}\right) \delta$. If $a \in A, \xi, \eta \in H, f, g, h, k \in C_{00}(G)$ we have:

$$
\begin{aligned}
& \langle(\delta \otimes i) \delta(a)(\xi \otimes f \otimes g), \eta \otimes h \otimes k\rangle \\
& =\int\left\langle\delta(a)\left(\mu\left(f_{t}\right) \otimes I\right)(\xi \otimes g),\left(\mu\left(h_{t}\right) \otimes I\right)(\eta \otimes k)\right\rangle d t \\
& =\int\left\langle\delta(a)\left(\mu\left(f_{t}\right) \xi \otimes g\right), \mu\left(h_{t}\right) \eta \otimes k\right\rangle d t \\
& =\iint\left\langle a \mu\left(g_{s} \cdot f_{t}\right) \xi, \mu\left(k_{s} \cdot h_{t}\right) \eta\right\rangle d t d s \\
& =\iint\left\langle a \mu\left(g_{s t} \cdot f_{s}\right) \xi, \mu\left(k_{s t} \cdot h_{s}\right) \eta\right\rangle d s d t \\
& =\int\left\langle\delta(a)\left(\xi \otimes g_{t} f\right), \eta \otimes k_{t} h\right\rangle d t \\
& =\int\left\langle\delta(a)\left(I \otimes g_{t}\right)(\xi \otimes f),\left(I \otimes k_{t}\right)(\eta \otimes h)\right\rangle d t
\end{aligned}
$$

(Lemma 2)

$$
\begin{aligned}
& =\left\langle W^{*}(\delta(a) \otimes I) W(\xi \otimes f \otimes g), \eta \otimes h \otimes k\right\rangle \\
& =\left\langle\left(i \otimes \delta_{G}\right) \delta(a)(\xi \otimes f \otimes g), \eta \otimes h \otimes k\right\rangle .
\end{aligned}
$$

We have now shown that $(A, \delta)$ is a $\mathscr{L}(G)$-comodule, and in order to complete the proof of Theorem 1 we shall show that $\delta$ defined in (27) is an isomorphism between the covariant systems $(\mathfrak{H}, \varrho, G)$ and $\left(W^{*}(A, \delta, G), \varrho^{\sim}, G\right)$ where $\varrho^{\sim}$ is the natural automorphic representation of $G$ on $W^{*}(A, \delta, G)$ defined by (9).

If we put an element $\mu(h)$ with $h \in L^{\infty}(G)$ into (27) we have from (23) that

$$
\begin{aligned}
& \langle\delta(\mu(h))(\xi \otimes f), \eta \otimes g\rangle=\left\langle p\left(\mu(g)^{*} \mu(h) \mu(f)\right) \xi, \eta\right\rangle \\
& =\langle\xi, \eta\rangle\langle h f, g\rangle=\langle(I \otimes h)(\xi \otimes f), \eta \otimes g\rangle .
\end{aligned}
$$

Thus $\delta(\mu(h))=I \otimes h$ and from Lemma 4 it follows that $\delta(\mathfrak{U})=W^{*}(A, \delta, G)$. From (28) it follows that

$$
\varrho_{x}^{\sim}(\delta(a))=(I \otimes v(x)) \delta(a)\left(I \otimes v\left(x^{-1}\right)\right)=\delta\left(\varrho_{x}(a)\right) \text { for } \quad x \in G, a \in \mathfrak{A},
$$

proving that the covariant systems $(\mathfrak{A}, \varrho, G)$ and $\left(W^{*}(A, \delta, G), \varrho \sim, G\right)$ are equivalent.

\section{§3. The Bidual of a Covariant System and of a $\mathscr{L}(G)$-Comodule}

We have now seen that a $\mathscr{L}(G)$-comodule $(A, \delta)$ gives rise to a covariant system $(\mathfrak{U}, \varrho, G)$ with $\mathfrak{U}=W^{*}(A, \delta, G)$. In [2] it was shown that a covariant system $(A, \varrho, G)$ gives rise to a $\mathscr{L}(G)$-comodule $(\mathfrak{U}, \delta)$ with $\mathfrak{U}=W^{*}(A, \varrho, G)$. It is therefore natural to ask what $W^{*}\left(W^{*}(A, \varrho, G), \delta, G\right)$ and $W^{*}\left(W^{*}(A, \delta, G), \varrho, G\right)$ are. It should come as no surprise that both are isomorphic to $A \otimes \mathscr{B}\left(L^{2}(G)\right)$, a fact which was proved for an abelian $G$ in [8].

Theorem 2. Given a covariant system $(A, \varrho, G)$ let $(\mathfrak{U}, \delta)$ be the $\mathscr{L}(G)$-comodule defined in $\left[2\right.$, Chapter 2], i.e. $\mathfrak{U}=W^{*}(A, \varrho, G)$ and $\delta(a)=W^{*}(a \otimes I) W$. Then $W^{*}(\mathfrak{U}, \delta, G) \cong A \otimes \mathscr{B}\left(L^{2}(G)\right)$.

Proof. Suppose $A$ acts on a Hilbert space $H_{0}$ and let $\varrho^{\sim}$ be the faithful representation of $A$ on $H=L^{2}\left(G, H_{0}\right)$ given by

$$
\varrho(a) f(s)=\varrho_{s^{-1}}(a) f(s) \text { for } a \in A, f \in H, s \in G .
$$


Then $\mathfrak{A}=\left[\varrho^{\sim}(A) \cup I \otimes \mathscr{L}(G)\right]^{\prime \prime}$ and $\delta(\mathfrak{A})$ is generated by $\varrho^{\sim}(A) \otimes I \cup\{I \otimes x \otimes x \mid x \in G\}$. So $W^{*}(\mathfrak{A}, \delta, G)$ is generated over $L^{2}\left(G \times G, H_{0}\right)$ by

$\varrho^{\sim}(A) \otimes I \cup I \otimes \delta_{G}(\mathscr{L}(G)) \cup I \otimes I \otimes L^{\infty}(G)$.

Let $\varrho^{0}: G \rightarrow$ Aut $\varrho^{\Upsilon}(A)$ be given by

$$
\varrho_{x}^{0}(a)=(I \otimes x) a\left(I \otimes x^{-1}\right) \quad \text { for } \quad x \in G, a \in \varrho^{\sim}(A) .
$$

The covariant systems $\left(\varrho^{\sim}(A), \varrho^{0}, G\right)$ and $(A, \varrho, G)$ are then equivalent. From [2, Proposition 2.2] it follows that

$$
\varrho^{\sim}(A) \otimes \mathscr{B}\left(L^{2}(G)\right)=\left[W^{*}\left(\varrho^{\sim}(A), \varrho^{0}, G\right) \cup I \otimes I \otimes L^{\infty}(G)\right]^{\prime \prime} .
$$

Let $U$ be the unitary operator over $L^{2}\left(G \times G, H_{0}\right)$ defined by

$$
U f(s, t)=f\left(t^{-1} s, t\right) .
$$

Then

$$
W^{*}\left(\varrho^{\sim}(A), \varrho^{0}, G\right)=\left[U^{*}\left(\varrho^{\sim}(A) \otimes I\right) U \cup I \otimes I \otimes \mathscr{L}(G)\right]^{\prime \prime} .
$$

So

$$
\varrho^{\sim}(A) \otimes \mathscr{B}\left(L^{2}(G)\right)=\left[U^{*}\left(\varrho^{\sim}(A) \otimes I\right) U \cup I \otimes I \otimes \mathscr{L}(G) \cup I \otimes I \otimes L^{\infty}(G)\right]^{\prime \prime} .
$$

Now $U(I \otimes I \otimes x) U^{*}=I \otimes x \otimes x$ for $x \in G$, and $U \in\left(I \otimes I \otimes L^{\infty}(G)\right)^{\prime}$ so

$$
A \otimes \mathscr{B}\left(L^{2}(G)\right) \cong \varrho^{\sim}(A) \otimes \mathscr{B}\left(L^{2}(G)\right)=U^{*} W^{*}(\mathfrak{A}, \delta, G) U,
$$

which proves the theorem.

Theorem 3. Given a $\mathscr{L}(G)$-comodule $(A, \delta)$ let $(\mathfrak{A}, \varrho, G)$ be the covariant system defined by $\mathfrak{A}=W^{*}(A, \delta, G)$ and $\varrho$ as in (9). Then $W^{*}(\mathfrak{H}, \varrho, G) \cong A \otimes \mathscr{B}\left(L^{2}(G)\right)$.

Proof. $\mathfrak{A}=W^{*}(A, \delta, G)$ is generated by $\delta(A) \cup\left(I \otimes L^{\infty}(G)\right)$ over $L^{2}\left(G, H_{0}\right)$ if we consider $A$ as a von Neumann algebra over $H_{0} . B=W^{*}(\mathfrak{U}, \varrho, G)$ is then generated by $V^{*}(\mathfrak{A} \otimes I) V \cup I \otimes I \otimes \mathscr{L}(G)$ where $V$ is the unitary operator over $L^{2}\left(G \times G, H_{0}\right)$ defined by

$$
V f(s, t)=\Delta(t)^{1 / 2} f(s t, t) \text {. }
$$

Define another unitary operator $S$ by

$$
S f(s, t)=\Delta(t)^{-1 / 2} f\left(s, t^{-1}\right)
$$

then $S^{*} V^{*}(I \otimes \varphi \otimes I) V S=W(I \otimes I \otimes \varphi) W^{*}$ for $\varphi \in L^{\infty}(G)$, and $S^{*}(I \otimes I \otimes x) S=$ $I \otimes I \otimes v(x)$ for $x \in G$. Since $V S$ and $\delta(A) \otimes I$ commute we therefore have that

$$
\begin{aligned}
B & =\left[V^{*}(\delta(A) \otimes I) V \cup V^{*}\left(I \otimes L^{\infty}(G) \otimes I\right) V \cup I \otimes I \otimes \mathscr{L}(G)\right]^{\prime \prime} \\
& =S\left[\delta(A) \otimes I \cup W\left(I \otimes I \otimes L^{\infty}(G)\right) W^{*} \cup I \otimes I \otimes \mathscr{R}(G)\right]^{\prime \prime} S^{*} \\
& =S W\left[W^{*}(\delta(A) \otimes I) W \cup I \otimes I \otimes L^{\infty}(G) \cup I \otimes I \otimes \mathscr{R}(G)\right]^{\prime \prime} W^{*} S^{*} .
\end{aligned}
$$

So if we can prove that

$$
\left[W^{*}(\delta(A) \otimes I) W \cup I \otimes I \otimes \mathscr{B}\left(L^{2}(G)\right)\right]^{\prime \prime}=\delta(A) \otimes \mathscr{B}\left(L^{2}(G)\right)
$$


the theorem is proved. (38) is equivalent to

$$
W^{*}\left(\delta(A)^{\prime} \otimes \mathscr{B}\left(L^{2}(G)\right)\right) W \cap\left(I \otimes I \otimes L^{\infty}(G)\right)^{\prime} \cap(I \otimes I \otimes \mathscr{R}(G))^{\prime}=\delta(A)^{\prime} \otimes I,
$$

i.e. that

$$
W^{*}\left(\delta(A)^{\prime} \otimes \mathscr{L}(G)\right) W \cap\left(I \otimes I \otimes L^{\infty}(G)\right)^{\prime}=\delta(A)^{\prime} \otimes I .
$$

Let $D=W\left(I \otimes I \otimes L^{\infty}(G)\right) W^{*}$ and $E=\left(\delta(A)^{\prime} \otimes \mathscr{L}(G)\right) \cap D^{\prime}$. Now define a map $\delta^{\prime \prime}: E \rightarrow E \otimes \mathscr{L}(G)$ by

$$
\delta^{\prime \prime}(a)=\left(I \otimes I \otimes W_{G}^{*}\right)(a \otimes I)\left(I \otimes I \otimes W_{G}\right)=\left(i \otimes \delta_{G}\right)(a) \quad \text { for } \quad a \in E,
$$

where $i$ is the identity automorphism of $\mathscr{B}\left(L^{2}\left(G, H_{0}\right)\right)$. Obviously $\delta^{\prime \prime}\left(\delta(A)^{\prime} \otimes\right.$ $\mathscr{L}(G)) \subset \delta(A)^{\prime} \otimes \mathscr{L}(G) \otimes \mathscr{L}(G)$ and $\delta^{\prime \prime}(D)=D \otimes I$, so (41) will in fact define a normal isomorphism of $E$ into $E \otimes \mathscr{L}(G)$ which obviously satisfies $\left(\delta^{\prime \prime} \otimes i\right) \delta^{\prime \prime}=\left(i \otimes \delta_{G}\right) \delta^{\prime \prime}$. $I \otimes v(x) \otimes x=W(I \otimes v(x) \otimes I) W^{*} \in E$ and $\delta^{\prime \prime}(I \otimes v(x) \otimes x)=I \otimes v(x) \otimes x \otimes x$ for $x \in G$, so again we can use Theorem 1 in [2] to conclude that $E$ is generated by

$$
F=\left\{a \in E \mid \delta^{\prime \prime}(a)=a \otimes I\right\} \quad \text { and } \quad\{I \otimes v(x) \otimes x \mid x \in G\} .
$$

Using the same argument as in (21) we have that

$$
\begin{aligned}
F & =E \cap\left(I \otimes I \otimes L^{\infty}(G)\right)^{\prime}=\left(\delta(A)^{\prime} \otimes \mathscr{L}(G)\right) \cap D^{\prime} \cap\left(I \otimes I \otimes L^{\infty}(G)\right)^{\prime} \\
& =\left(\delta(A)^{\prime} \otimes \mathscr{L}(G)\right) \cap\left(I \otimes L^{\infty}(G) \otimes L^{\infty}(G)\right)^{\prime} \\
& =\left(\delta(A)^{\prime} \cap\left(I \otimes L^{\infty}(G)\right)^{\prime}\right) \otimes I=C \otimes I
\end{aligned}
$$

where $C$ is as in (20). So the left hand side of (40) equals:

$$
\begin{aligned}
W^{*} E W & =W^{*}[F \cup\{I \otimes v(x) \otimes x \mid x \in G\}]^{\prime \prime} W \\
& =[C \otimes I \cup I \otimes \mathscr{R}(G) \otimes I]^{\prime \prime}=\delta(A)^{\prime} \otimes I
\end{aligned}
$$

according to (19). So the formula (40) holds and the theorem is proved.

\section{§4. The Commutant of a Covariance Algebra and of a Dual Covariance Algebra}

Digernes proved in [1, Theorem 3.14] the following:

Theorem 4. Suppose $A$ is a von Neumann algebra over a Hilbert space $H$ and that $U$ is a continuous unitary representation on $H$ of the locally compact group $G$ such that

$$
\varrho_{x}(a)=U_{x} a U_{x^{-1}} \in A \quad \text { for all } \quad x \in G, a \in A .
$$

Let the covariance algebra $\mathfrak{U}=W^{*}(A, \varrho, G)$ act on $L^{2}(G, H)$ as usual. Then $\mathfrak{U}^{\prime}$ is generated by $A^{\prime} \otimes I$ and $\left\{U_{x} \otimes v(x) \mid x \in G\right\}$, and in fact $\mathfrak{U}^{\prime} \cong W^{*}\left(A^{\prime}, \varrho^{\prime}, G\right)$ where $\varrho^{\prime}: G \rightarrow \operatorname{Aut}\left(A^{\prime}\right)$ is defined by

$$
\varrho_{x}^{\prime}(a)=U_{x} a U_{x^{-1}} \quad \text { for } \quad a \in A^{\prime} \text {. }
$$

We shall first give an alternate proof of this theorem using [2, Theorem 1] and then state and prove a similar result for the dual covariance algebra of a $\mathscr{L}(G)$-comodule. 
Proof of Theorem 4. Let $\mathfrak{U}=W^{*}(A, \varrho, G)$ as defined in [2], then

$$
W\left(\mathfrak{U}^{\prime} \otimes I\right) W^{*} \subset \mathfrak{U}^{\prime} \otimes \mathscr{L}(G),
$$

cf. the first part of the proof of Lemma 1, and it is straight forward to check that

$$
U_{x} \otimes v(x) \in \mathfrak{U}^{\prime} \text { for } \quad x \in G .
$$

Defining the map $\delta^{\prime}$ as in (16), i.e.

$$
\delta^{\prime}(a)=W(a \otimes I) W^{*} \text {, for } a \in \mathfrak{X}^{\prime}
$$

we have that $\delta^{\prime}\left(U_{x} \otimes v(x)\right)=U_{x} \otimes v(x) \otimes x$ and that $\left(\delta^{\prime} \otimes i\right) \delta^{\prime}=\left(i \otimes \delta_{G}\right) \delta^{\prime}$, so as in Lemma 1 we can use [2, Theorem 1] to conclude that $\mathfrak{I}^{\prime}$ is generated by

$$
C \cup\left\{U_{x} \otimes v(x) \mid x \in G\right\}
$$

where

$$
C=\left\{c \in \mathfrak{U}^{\prime} \mid \delta^{\prime}(c)=c \otimes I\right\} .
$$

For $C$ we can use the same argument as in (21) to conclude that $C=\mathfrak{U}^{\prime} \cap\left(I \otimes L^{\infty}(G)\right)^{\prime}$, so $C=A^{\prime} \otimes I$ according to [2, Proposition 2.2].

So $\mathfrak{U}^{\prime}=\left[A^{\prime} \otimes I \cup\left\{U_{x} \otimes v(x) \mid x \in G\right\}\right]^{\prime \prime}$ as stated, furthermore, Theorem 1 of [2] also gives us that $\mathfrak{U}^{\prime} \cong W^{*}\left(A^{\prime}, \varrho^{\prime}, G\right)$.

The dual version of Theorem 4 is the following:

Theorem 5. Suppose $(A, \delta)$ is a $\mathscr{L}(G)$-comodule and let $\mathfrak{A}=W^{*}(\delta(A), \delta \otimes i, G)$ be the covariance algebra of the equivalent comodule $(\delta(A), \delta \otimes i)$. Then

$$
\mathfrak{U}^{\prime}=\left[\delta(A)^{\prime} \otimes I \cup W^{*}\left(I \otimes I \otimes L^{\infty}(G)\right) W\right]^{\prime \prime}
$$

and $\mathfrak{U}^{\prime}$ is isomorphic to the covariance algebra of the $\mathscr{L}(G)$-comodule $\left(\delta(A)^{\prime}, \delta^{\prime}\right)$ where $\delta^{\prime}$ is defined by

$$
\delta^{\prime}(a)=W(a \otimes I) W^{*} \quad \text { for } \quad a \in \delta(A)^{\prime} .
$$

Proof. $\mathfrak{A}=\left[W^{*}(\delta(A) \otimes I) W \cup I \otimes I \otimes L^{\infty}(G)\right]^{\prime \prime} .(I \otimes I \otimes v(x)) \mathfrak{U}^{\prime}\left(I \otimes I \otimes v\left(x^{-1}\right)\right)=\mathfrak{A}^{\prime}$ and if we define $\mu: L^{\infty}(G) \rightarrow \mathfrak{U}^{\prime}$ by

$$
\mu(f)=W^{*}(I \otimes I \otimes f) W \quad \text { for } \quad f \in L^{\infty}(G)
$$

we see that with $\varrho$ as in (9), Theorem 1 is satisfied so $\mathfrak{U}^{\prime}$ is generated by $\mu\left(L^{\infty}(G)\right)$ and $\mathfrak{U}^{\prime} \cap(I \otimes I \otimes v(G))^{\prime}$. From (40) we have that

$$
\begin{aligned}
\mathfrak{I}^{\prime} \cap(I \otimes I \otimes v(G))^{\prime} & =\left[W^{*}(\delta(A) \otimes I) W \cup I \otimes I \otimes L^{\infty}(G) \cup I \otimes I \otimes v(G)\right]^{\prime} \\
& =\delta(A)^{\prime} \otimes I .
\end{aligned}
$$

This proves (43). Furthermore

$$
\begin{aligned}
W \mathfrak{I}^{\prime} W^{*} & =\left[W\left(\delta(A)^{\prime} \otimes I\right) W^{*} \cup I \otimes I \otimes L^{\infty}(G)\right]^{\prime \prime} \\
& =W^{*}\left(\delta(A)^{\prime}, \delta^{\prime}, G\right)
\end{aligned}
$$

where $\delta^{\prime}$ is defined by (44). It was proved in Lemma 1 that $\left(\delta(A)^{\prime}, \delta^{\prime}\right)$ really is a $\mathscr{L}(G)$-comodule. 